\title{
“At Least Your Baby Is Healthy" Obstetric Violence or Disrespect and Abuse in Childbirth Occurrence Worldwide: A Literature Review
}

\author{
Violette Perrotte ${ }^{1}$, Arun Chaudhary ${ }^{1}$, Annekathryn Goodman ${ }^{2 *}$ \\ ${ }^{1}$ Massachusetts General Hospital, Boston, USA \\ ${ }^{2}$ Department of Obstetrics and Gynecology Yawkey, Boston, Massachusetts, USA \\ Email: violetteperrotte@gmail.com, arun.ac0806@gmail.com, *agoodman@mgh.harvard.edu
}

How to cite this paper: Perrotte, V., Chaudhary, A. and Goodman, A. (2020) "At Least Your Baby Is Healthy" Obstetric Violence or Disrespect and Abuse in Childbirth Occurrence Worldwide: A Literature Review. Open Journal of Obstetrics and Gynecology, 10, 1544-1562.

https://doi.org/10.4236/ojog.2020.10110139

Received: October 4, 2020

Accepted: November 13, 2020

Published: November 16, 2020

Copyright $\odot 2020$ by author(s) and Scientific Research Publishing Inc. This work is licensed under the Creative Commons Attribution International License (CC BY 4.0).

http://creativecommons.org/licenses/by/4.0/

\begin{abstract}
Obstetric violence or disrespect and abuse in childbirth is a worldwide phenomenon that takes on various forms, from absence of informed consent to physical harm. The objective of this review is to assess prevalence and root causes of obstetric violence in different countries, and potential solutions to address disrespect and abuse in childbirth. The review finds that obstetric violence is rooted in a patriarchal understanding of gender stereotypes and is exacerbated by power dynamics between health professionals and patients, especially for minorities. Obstetric violence has a long-lasting impact on women's lives and can jeopardize subsequent decisions to access healthcare services.
\end{abstract}

\section{Keywords}

Obstetric Violence, Disrespect and Abuse, Childbirth, Women

\section{Introduction}

The most commonly used definition of obstetric violence (OV) comes from Bowser and Hill's seven categories of disrespect and abuse (D \& A): physical abuse, non-consensual care, non-confidential care, non-dignified care, discrimination based on specific patient attribute, abandonment of care, and detention in facilities [1] [2]. Violent acts in childbirth can take various forms, from joking about a patient's pain, to forced sterilization after delivery [3]-[9]. Table 1 gives examples of such acts. Disrespect and abuse have been used as an alternative term to OV [10]. 
Table 1. Categories of obstetrical violence as defined by Bowser and Hill [1].

\begin{tabular}{|c|c|}
\hline $\begin{array}{c}\text { Categories of } \\
\text { Obstetrical Violence }\end{array}$ & Examples \\
\hline 1. Physical Abuse & $\begin{array}{ll}\text { - } & \text { Slap during delivery } \\
\text { - } & \text { Physically restrain during delivery } \\
\text { - } & \text { Perform unnecessary procedures }[12]\end{array}$ \\
\hline 2. Non-Consensual Care & $\begin{array}{l}\text { Impose temporary or permanent contraception after delivery, } \\
\text { or forced C-section for non-vital medical reasons [2] } \\
\text { - Patient's permission before performing a procedure not asked }\end{array}$ \\
\hline 3. Non-Confidential Care & - Absence of curtains or a blanket during examinations [4] \\
\hline 4. Non-Dignified Care & $\begin{array}{l}\text { - Force patients to deliver in a supine position even if } \\
\text { not necessary [14] } \\
\text { - Joking about patient's pain }\end{array}$ \\
\hline 5. Discrimination & $\begin{array}{l}\text { - } \quad \text { Talk in a language that patients do not understand [15] } \\
\text { Allow medical students to perform unnecessary medical } \\
\text { procedures on patients in public facilities to learn, while } \\
\text { refraining to do so for patients with private healthcare [7] }\end{array}$ \\
\hline 6. Abandonment of Care & $\begin{array}{l}\text { - Deliver without skilled birth attendants } \\
\text { - Discharge patients against their will if refuse recommended } \\
\text { treatment }\end{array}$ \\
\hline 7. Detention in Facilities & Detain in facility for failure to pay \\
\hline
\end{tabular}

The broad definition of $\mathrm{D} \& \mathrm{~A}$ derives from the various forms it takes in different countries and cultures. The spectrum of violent acts that take place in birth and delivery are specific to each country's culture and context and can be associated with both hyper- and under-medicalization [6] [11] [12] [13]. For example, in the Americas and Europe, forced caesarean sections and episiotomies are very frequent (hyper-medicalization), whereas in low- and middle-income countries, unattended delivery in a health center is more likely (under-medicalization).

Abuya et al. [3] have attempted to organize Bowser and Hill's categories of violence into experiential building blocks, where violent acts occur in childbirth "regardless of patient experience or provider intention" and normative building blocks, where behaviors that depart from a set of standard codes and behaviors are considered violations. Experiential building blocks ensure that patient perspective is at the center of defining what constitutes violence, while using these experiences to reframe violent acts as objective events. Normative building blocks rely on notions of "human rights law, domestic law, ethical codes, and local consensus on behaviors" to anchor obstetrical violence in structural discrimination and violation of community codes [3] [13].

At the center of the discussion on obstetric violence is the evolution of maternal health metrics. Health metrics include subjective assessments of quality of life from the patient's perspective [16]. Where public health authorities once only looked at outcomes of births, the conversation is now shifting towards the process of birth. Absence of maternal and fetal deaths no longer constitutes the sole marker of a high-quality maternal health system [5] [13]. The World Health 
Organization and the International Consortium for Health Outcomes Measurement recognize women's experience of maternal healthcare as a crucial determinant of health outcomes and recommend including it in maternal health metrics [10] [17]. The process of healthcare is equally important as the structure of the health system for health care quality and outcome [1] [10] [17] [18]. The WHO also advocates "people-centeredness" as an important characteristic of quality of care, which takes the service user's preference into account [10].

Obstetric violence has most likely occurred throughout human history and was embedded in the development of obstetrics and gynecology as a specialty. For instance, James Marion Sims used enslaved black women to test out the use of the speculum without anesthesia or consent in the $19^{\text {th }}$ Century [19]. However, D \& A in childbirth was only recently put at the forefront of violence against women (VAW), due to social media campaigns and viral hashtags (\#PayeTonUtérus in France, \#PrekinimoŠutnju in Croatia, \#Metoointhebirthroom in England, \#violence_in_obstetrics in Russia, \#violenciaobstetrica in Central America, \#SomosTodasAdelir in Brazil, \#DiLaVerdadAnaRoman in Chile) that offer women a platform to speak out about their traumatic childbirth experience [6] [20].

The goal of the obstetric violence movement is to reframe seemingly routine medical acts into violation of women's sexual and reproductive rights [12]. The gendered nature of OV puts women at the center of the violent acts, "because of who they are (female reproducers) and what they represent (a risk to high mortality figures)" [21]. Because victims of OV are always female patients, some scholars categorize OV as a form of Gender-Based Violence [22]. Women are seen as the vector to the delivery of a healthy child, in a context where the main objective is to keep both patients alive at all costs.

The medical sphere has overall privileged the notion of "obstetric logic" [21] to justify certain delivery procedures that patients, on the other hand, experience as "birth rape" [14]. The violence is then justified by "well-meaning physicians" [12] who execute the delivery in conditions they deem necessary, even if women's rights are violated in the process [13] [14] [23].

Common to all forms of $\mathrm{OV}$ are its roots in institutional violence and discrimination against women. Because OV often entails a clinical setting, the health center or hospital where it takes place becomes the institution of violence, and the absence of accountability or criminalization mechanisms in many countries reinforces this overwhelming institutional power that the medical profession holds over patients. Patients of low socio-economic status (SES) and cultural and religious minorities are especially vulnerable [7] [21] [24] [25].

Obstetric violence is rooted in a system of multifold power hierarchies, providers versus (vs.) patients, physicians vs. midwives, minorities vs. dominant race, low SES women vs. women with private health insurance. These power dynamics contribute to creating an enabling environment for obstetric violence to take place and reinforce the infantilization of the female patient into an inferior being, expected to submit to medical forces and ignore their laboring bodily 
autonomy [3] [7] [15] [25].

The absence of protection for women during birth and in cases of legal action against OV reflects a failure to recognize violence when it is not "codified and regulated" and is structurally embedded within institutions [12]. The lack of consensus on what constitutes obstetric violence correlates with a dearth of screening tools, making it virtually impossible for women to take legal action, even for physically violent forms of OV. Only Latin American countries have criminalized obstetrical violence by clearly stating in federal laws what obstetrical incidents are legally reprehensible [11] [12] [23].

This study aims to understand the prevalence and roots of obstetric violence, differences in instances by regions of the world, and existing and potential solutions to reduce harm in childbirth. Obstetric violence is universal but takes many forms based on economic context, level of medicalization, composition of healthcare teams, and perception of gender in culture. The diversity of approaches to examine obstetric violence makes a literature review of worldwide publications imperative. This study will foster a more comprehensive view of the phenomenon of disrespect and abuse in childbirth, in the hopes of informing future interventions and policies.

\section{Methods}

This study used an Integrative Literature Review methodology to evaluate publications related to violence in childbirth and traumatic obstetrical experiences. The Integrative Literature Review method was chosen for its ability to include different methodologies both experimental and non-experimental, and its broad inclusion of literature, which offers a more comprehensive approach to the topic of obstetrical violence [26]. Relevant publications were reviewed and synthesized in a taxonomy of constructs [27], to analyze existing findings and generate new information in the field of obstetric violence. This synthesis allowed for an overview of the existing knowledge and approaches around OV by regions of the world.

Keywords used to conduct the literature search were "obstetrical violence", "violence in childbirth", "traumatic childbirth", "gender-based violence", "disrespect and abuse", and "violences obstétricales". The meaning of these key words follows the WHO definition [28]. Abstracts were read to identify inclusion criteria, and selected publications were read entirely. Search engines and online libraries used were: Hollis (Harvard University Library Search Engine), BioMedical Central (BMC), National Center for Biotechnology Information (NCBI), PubMed (U.S. National Library of Medicine National Institute of Health), le Haut Conseil à l'Egalité entre les Femmes et les Hommes (HCE), and l'Institut de Recherche et d'Actions pour la Santé des Femmes (IRASF).

Inclusion criteria were: Qualitative and quantitative studies in the English or French languages, published from 2015 through 2020, which focused on questions of the prevalence of obstetric violence, Gender-Based Violence in childbirth, roots of obstetric violence, solutions to obstetric violence, and obstetric violence in legal frameworks, in all countries of the world (and translated if neces- 
sary). Exclusion criteria were: Publications written before 2015 and journalistic pieces. The time span of this study was selected to complement a recent literature review focused on obstetric violence in the daily routine of care, which looked at literature from 2007 to 2017 [29], and because our study encompasses the large number of papers published between 2015 and 2020, following newfound public attention to the issue of obstetric violence. Figure 1 shows the paper selection, identification, and inclusion of publications for this review. Given that this was a descriptive review of the current literature, sample size estimations and statistics were not done.

A total of 22 publications were selected, read in full length, organized by regions of the world and broken down into the following categories: Relevance, context, major findings, definition of obstetric violence, impact of obstetric violence (on victims and society), role of culture in fostering favorable environments for obstetric violence, physician incentives, mention of fetal life rights, and solutions and remaining challenges. All selected literature addresses obstetric violence from a different angle, and provides various perspectives, including physicians', with a broader analysis of the roots of obstetric violence in Gender-Based Violence.

\section{Results}

Although disrespect and abuse in childbirth take different forms and are expressed differently by women who experience it, it is found in every region of the world. Seven $(31.8 \%)$ of the reviewed publications were from Latin America, with a large majority from Mexico (five out of seven Latin American publications). Five publications (22.8\%) were from the African continent, $13.7 \%(n=3)$

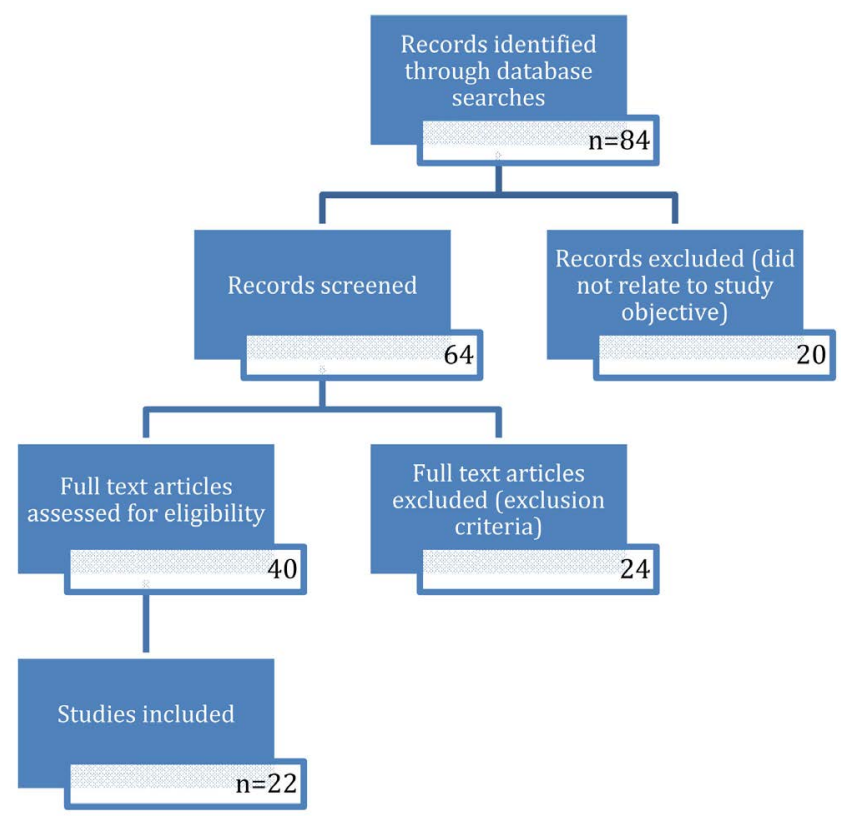

Figure 1. Identification, selection, and inclusion of publications on obstetrical violence from 2015 through 2020 for the integrative literature review. 
from the United States, 9\% $(n=2)$ from Europe (all from France), and 9\% $(n=$ 2) from Asia (one from Iran, and another from Sri Lanka). The remaining 13.7\% publications $(n=3)$ were literature reviews or philosophical papers, which either did not focus on any specific country or the geographical region was not relevant. Thirty-two percent of publications were published in 2015, when the $M e$ Too movement and related social media campaigns started, 9\% in 2016, $4.5 \%$ in $2017,27 \%$ in $2018,23 \%$ in 2019 and $4.5 \%$ in 2020 . Forty-one percent of the studies conducted were of qualitative nature, all studies interviewed or observed patients but only six included the perspectives of providers (27\%). Eighteen percent were quantitative. Table 2 and Table 3 highlight the details according to the seven Obstetric Violence categories of Bowser and Hill [1] for eight studies where complete information was available [2] [25].

\section{North America}

According to a 2015 household survey in Mexico $(n=24,064), 23.6 \%$ of women aged 15 - 49 years old who had given birth in the previous five years had experienced some form of obstetric violence [2]. The same study found that $17.1 \%$ of women experienced unconsented care, $11.2 \%$ were yelled at, $7 \%$ were insulted, $17.1 \%$ were forced to deliver in an uncomfortable position, $9.9 \%$ had their questions ignored, and $10.3 \%$ had delayed care. The study also reported that women are 3.7 times more likely to experience OV at public hospitals compared to private hospital. Another Mexican study that surveyed women who received obstetric care at two public hospitals $(n=512)$ found that $29 \%$ of women had experienced some form of abuse [7].

A national US survey $(n=2138)$ documented that $17.3 \%$ of women who were pregnant between 2010 and 2016 experienced some form of OV [25]. More specifically, $5.1 \%$ of those who gave birth at home compared to $28.1 \%$ who gave birth at hospital experienced OV; $1.3 \%$ women reported physical abuse, $1.2 \%$ had private information shared without consent, $5.5 \%$ had physical privacy violated, $8.5 \%$ were shouted at, $4.5 \%$ were threatened that treatment would be withheld, and $7.8 \%$ had requests ignored, refused or delayed. Women with lower socioeconomic status (SES), and elevated pregnancy or social risks experienced more discriminatory care: $21.5 \%$ low SES patients compared to $15.0 \%$ higher SES patients, $27.9 \%$ of women with elevated pregnancy risk compared to $14.5 \%$ of lower pregnancy risk, and $30.1 \%$ of high social risk women compared to $16.1 \%$ lower risk group experienced some form of OV. In addition, racial discrimination lead to higher instances of OV: $22.5 \%$ of Black women, $25.0 \%$ of Hispanics, $32.8 \%$ of Indigenous women, $21.1 \%$ of Asian women, compared to $14.1 \%$ of White women reported having experienced some form of OV.

\section{Africa}

Lack of privacy was common in under-resourced countries in the African continent, with $58.2 \%$ of women reporting not being given any privacy during their birth in Malawi [8], and 8.5\% in Kenya [3]. Non-dignified care was also reported in $8 \%$ of cases in Tanzania (Sando et al., 2016) and 8.5\% in Kenya [3]. 
Table 2. Prevalence (\%) of obstetric violence by categories $1-4[1]$.

\begin{tabular}{|c|c|c|c|}
\hline Country & $\begin{array}{l}\text { Overall prevalence of Ob- } \\
\text { stetric Violence }\end{array}$ & 1. Physical Abuse & $\begin{array}{l}\text { 2. Non-consensual care } \\
\text { 3. Non-confidential care } \\
\text { 4. Non-dignified Care }\end{array}$ \\
\hline Mexico [2] & $23.6 \%$ & $\begin{array}{l}9.2 \% \text { forced to stay } \\
\text { in uncomfortable } \\
\text { positions }\end{array}$ & $\begin{array}{l}17.1 \% \text { unconsented } \\
11.2 \% \text { yelled at or scolded } \\
7 \% \text { insults or humiliations }\end{array}$ \\
\hline USA [25] & $\begin{array}{l}\text { Overall: } 17.3 \% \\
\text { Home births: } 5.1 \% \\
\text { Hospital births: } 28.1 \%\end{array}$ & $1.3 \%$ & $\begin{array}{l}1.2 \% \text { private info shared } \\
5.5 \% \text { non-confidential } \\
8.5 \% \text { shouted at } \\
4.5 \% \text { threatened to } \\
\text { withhold treatment }\end{array}$ \\
\hline Mozambique [5] & $\begin{array}{l}\text { District 28\% } \\
\text { Central } 5 \%\end{array}$ & $\begin{array}{l}\text { District } 6.42 \% \\
\text { Central } 1.3 \%\end{array}$ & $\begin{array}{l}\text { District } 43.5 / 40.83 / 28.44 \\
\text { Central 0.7/0.3/13.2 }\end{array}$ \\
\hline Malawi [8] & $58 \%$ & $\begin{array}{l}\text { Hit, slapped, } \\
\text { pinched } 0.2 \%\end{array}$ & $\begin{array}{l}20.5 \% \text { exams } \\
58.2 \% \text { lack of privacy } \\
1.9 \% \text { threatened }\end{array}$ \\
\hline Tanzania $[13]^{*}$ & $\begin{array}{l}\text { Postpartum } 15 \% \\
\text { Community Follow-up: } 70 \%\end{array}$ & $\begin{array}{l}\text { Postpartum 5\% } \\
\text { Community } \\
\text { Follow-up: 52\% }\end{array}$ & $\begin{array}{l}\text { Postpartum } 6 \% \\
\text { Community Follow-up: } \\
53 \%\end{array}$ \\
\hline Kenya [3] & $20 \%$ & $4.3 \%$ & $\begin{array}{l}4.3 \% \\
8.5 \% \\
18 \%\end{array}$ \\
\hline Ethiopia [4] & $78.6 \%$ & $32.9 \%$ & $\begin{array}{l}94.8 \% \\
21.4 \% \\
12.1 \%\end{array}$ \\
\hline Ghana [30] & $34 \%$ & $6.2 \%$ & $\begin{array}{l}20.3 \% \text { c-section } \\
29.2 \% \text { vag exam } \\
34 \% \text { verbal abuse }\end{array}$ \\
\hline Guinea [30] & $30 \%$ & $19.3 \%$ & $\begin{array}{l}7.9 \% \text { c-section } \\
49.5 \% \text { vag exam } \\
26.9 \% \text { verbal abuse }\end{array}$ \\
\hline Nigeria [30] & $44 \%$ & $16 \%$ & $\begin{array}{l}4.5 \% \text { c-section } \\
67.2 \% \text { vag exam } \\
44.2 \% \text { verbal abuse }\end{array}$ \\
\hline Myanmar $[30]^{* *}$ & $18 \%$ & $3.3 \%$ & $\begin{array}{l}7.9 \% \text { c-section } \\
25.9 \% \text { vag exam } \\
18.4 \% \text { verbal abuse }\end{array}$ \\
\hline
\end{tabular}

${ }^{\star}$ Based on interviews immediately postpartum $\mathrm{n}=1914$ and at home in follow-up $\mathrm{n}=64$; ${ }^{\star *}$ Community survey data as observational data not available in Myanmar.

A Kenyan study of thirteen health facilities $(\mathrm{n}=641)$ reported an overall OV prevalence of $20 \%$ [3]. Disrespect and abuse included non-confidential care (8.5\%), non-dignified care (18\%), neglect or abandonment of care (14.3\%), non-consensual care (4.3\%) physical abuse (4.2\%), and detainment for non-payment of fees (8.1\%).

Among, 173 patients who received care at four health care facilities in Addis Ababa, Ethiopia, 78\% reported having experienced some form of OV, which 
Table 3. Prevalence (\%) of obstetric violence by categories 5 - 7 (Bowser \& Hill, 2010).

\begin{tabular}{|c|c|c|c|}
\hline Country & 5. Discrimination & $\begin{array}{l}\text { 6. Abandonment of } \\
\text { care }\end{array}$ & $\begin{array}{l}\text { 7. Detention in } \\
\text { Facilities }\end{array}$ \\
\hline Mexico [2] & $\begin{array}{l}3.66 \text { times higher rate of } \mathrm{OV} \\
\text { in public vs. private hospital }\end{array}$ & $\begin{array}{l}10.3 \% \text { delayed care } \\
9.9 \% \text { questions ignored }\end{array}$ & $\mathrm{NA}^{* * *}$ \\
\hline USA [25] & $\begin{array}{l}\text { Low SES } \\
\text { Yes } 21.5 \% \\
\text { No } 15 \% \\
\text { Elevated pregnancy risk } \\
\text { Yes } 27.9 \% \\
\text { No } 14.5 \% \\
\text { High social risks } \\
\text { Yes } 30.1 \% \\
\text { No } 16.1 \% \\
\text { Racial disparity } \\
\text { Black } 22.5 \% \\
\text { Hispanic } 25 \% \\
\text { Asian } 21.1 \% \\
\text { Indigenous } 32.8 \% \\
\text { White } 14.1 \%\end{array}$ & $\begin{array}{l}7.8 \% \text { ignored, refused or } \\
\text { failed to respond to } \\
\text { request in reasonable time }\end{array}$ & NA \\
\hline Mozambique [5] & $\begin{array}{l}\text { District } 1.83 \% \\
\text { Central1.3\% }\end{array}$ & $\begin{array}{l}\text { District } 46.79 \% \\
\text { Central } 12.6 \%\end{array}$ & $\begin{array}{l}\text { District } 0 \\
\text { Central 1\% }\end{array}$ \\
\hline Malawi [8] & NA & $\begin{array}{l}55.9 \% \text { no pain relief } \\
12.4 \% \text { separated from } \\
\text { newborn after delivery }\end{array}$ & NA \\
\hline Tanzania $[13]^{*}$ & NA & $\begin{array}{l}\text { Postpartum } 8 \% \\
\text { Community } \\
\text { Follow-up: } 52 \%\end{array}$ & $2 \%$ \\
\hline Kenya [3] & $\mathrm{NA}$ & $14.3 \%$ & $8.1 \%$ \\
\hline Ethiopia [4] & $19.7 \%$ & $39.3 \%$ & $0.6 \%$ \\
\hline Ghana [30] & $3.7 \%$ & $47.1 \%$ no pain relief & $4.8 \%$ \\
\hline Guinea [30] & $1.4 \%$ & $31.6 \%$ no pain relief & $8.7 \%$ \\
\hline Nigeria [30] & $5.8 \%$ & $56.9 \%$ no pain relief & $1.1 \%$ \\
\hline Myanmar $[30]^{* *}$ & $1.7 \%$ & $8.3 \%$ no pain relief & $5.4 \%$ \\
\hline
\end{tabular}

${ }^{\star}$ Based on interviews immediately postpartum $n=1914$ and at home in follow-up $n=64 ;{ }^{* *}$ Community survey data as observational data not available in Myanmar; ${ }^{* * *}$ NA data not available.

included physical abuse (32.9\%), non-consensual care (94.8\%), non-confidential care $(21.4 \%)$, non-dignified care $(12.1 \%)$, discriminatory care $(19.7 \%)$, abandonment of care (39.3\%) and detention for non-payment (0.6\%) [4].

In Dar es Salaam, Tanzania, among 1914 women who gave postpartum interviews and 64 who were followed up, 15\% in postpartum interviews compared to $70 \%$ in follow-up interviews reported having experienced some form of OV [13]. For each category of OV, women reported higher OV prevalence in follow-up interviews than in postpartum interviews, indicating that women are less likely to perceive or report OV immediately after birth. A major limitation of this 
study is a potential selection bias introduced by inclusion of merely $3 \%$ of the original cohort in follow-up interviews.

In a study of one central and two district hospitals in Mozambique $(\mathrm{n}=572)$, across all the OV categories, women from the district hospitals with limited resources reported higher prevalence of $\mathrm{OV}$ than women treated at the central hospital [5]. The reported prevalence included physical abuse (district: $6.4 \%$ vs. central: $1.3 \%$ ), non-consensual care (district: $43.6 \%$ vs. central: $0.7 \%$ ), non-confidential care (district: $40.8 \%$ vs. central: $0.3 \%$ ), non-dignified care (district: $28.4 \%$ vs. central: $13.2 \%$ ), discriminatory care (district: $1.8 \%$ vs. central: $1.3 \%$ ), abandonment of care (district: $46.8 \%$ vs. central: $12.6 \%$ ) and detention for nonpayment (district: $0.0 \%$ vs. central: $1.0 \%$ ).

In a Malawian study that observed labor and delivery cases in 40 facilities ( $\mathrm{n}=$ 2109), the prevalence of non-consensual care ranged from $0.09 \%$ (unindicted post-delivery examination of uterus) to $73.9 \%$ (failure to ask for client's concerns), non-dignified care ranged from $1.9 \%$ (shouted at, insulted or threatened) to $13.9 \%$ (failure to respectfully greet), non-confidential care ranged from $2.4 \%$ (lack of private bed) to $73.1 \%$ (failure to cover woman's body during treatment), abandonment of care ranged from $11.6 \%$ (restriction of support person during birth) to $93.7 \%$ (failure to ask preferred position for delivery), and the physical abuse prevalence was $0.2 \%$ [8].

Another study that surveyed three healthcare facilities in four countries-Ghana $(n=836)$, Guinea $(n=644)$, Nigeria $(n=631)$ and Myanmar $(n=$ 631) - found that the prevalence of physical OV was $6.2 \%$ in Ghana, $19.3 \%$ in Guinea and $16.0 \%$ in Nigeria and 3.3\% in Myanmar [30]. In Ghana, the most prevalent forms of non-confidential and non-consensual cares were for vaginal examination (29.2\% and $46.9 \%$ respectively), and other prevalences include $34 \%$ of verbal abuse, $3.7 \%$ of stigma or discrimination, $47.1 \%$ denial of requested pain relief, 3.4\% unattended deliveries, and $4.8 \%$ detainment for nonpayment of fees. In Guinea, the most prevalent forms of non-confidential and nonconsensual cares were for vaginal examination (49.5\%) and episiotomy (73.1\%) respectively, and other prevalences include $26.9 \%$ of verbal abuse, $1.4 \%$ stigma or discrimination, $31.6 \%$ denial of requested pain relief, $0.4 \%$ of unattended delivery, and $8.7 \%$ of detainment for nonpayment of fees. In Nigeria, the most prevalent forms of non-confidential and non-consensual cares were both for vaginal examination (67.2\% and 56.9\% respectively), and other prevalence include $44.2 \%$ of verbal abuse, $5.8 \%$ of stigma or discrimination, $56.9 \%$ denial of requested pain relief, $3.0 \%$ of unattended delivery, and $5.4 \%$ of detainment for nonpayment of fee.

No current studies from Central and Northern Africa looked at the prevalence of disrespect and abuse.

\section{Asia}

For Myanmar $(n=631)$, the most prevalent forms of non-confidential and non-consensual cares were for vaginal examination (25.9\%) and episiotomy (66.4\%) respectively, and other prevalences include $18.4 \%$ of verbal abuse, $1.7 \%$ 
of stigma or discrimination, $8.3 \%$ denial of requested pain relief, $0.6 \%$ unattended deliveries, and $1.1 \%$ detainment for nonpayment of fees [30].

An Iranian study conducted in sixty-four health centers in Tabriz $(n=800)$ to determine predictors of traumatic birth experience found intrapartum factors such as length of stay in labor room, freedom to move during labor and select childbirth position, access to pain relief, and presence of companion to be significant predictors of trauma [31]. 37\% women described their birth as traumatic, with a $97.8 \%$ rate of episiotomy and $88.7 \%$ of women unable to choose their childbirth positions. In addition, although $65 \%$ of women reported having to stay in the labor room for longer than twelve hours, $59.9 \%$ were not allowed to move during labor.

\section{Europe}

In Europe too, violence in childbirth takes various forms. In France, one in five births results in an episiotomy, with $50 \%$ of episiotomies being unconsented [6]. In Italy, $21 \%$ of women experience either physical or verbal abuse during their pregnancy [6]. With $20 \%$ births involving episiotomy and $82 \%$ epidural use, often against woman's desire, the study also highlights an issue of hyper-medicalization of birth in high OEC (Observatory of Economic Complexity) countries.

\section{Discussion}

The definition of obstetric violence (OV) varies by context, so a consistent and universal legal definition has yet to be accepted in main legal texts apart from countries in South America [11] [32]. Health professionals at large disagree with the use of the term violence, emphasizing the notion of intent to harm, which is absent in certain contexts of disrespect and abuse in childbirth [22]. Using Bowser and Hill's seven categories of violence or WHO's five categories of OV (routine and unnecessary interventions on the mother or the infant, verbal abuse, humiliation or physical aggression, lack of material and inadequate facilities, practices performed by residents and professionals without consent, and discrimination on cultural, economic, religious and ethnic grounds) [29], the reviewed studies found incidences of violence in every region of the world.

All reviewed studies reveal that obstetric violence has a lasting impact on patients' willingness to use health services and seeking for postpartum care. Withdrawal from healthcare services not only applies to gynecological care, but to all other forms of medical services. If disrespect and abuse occur during the prenatal period, women may be discouraged to deliver in a facility, or voice concerns related to their pregnancies to their provider, potentially jeopardizing the safety of their delivery and subsequent care [4] [5] [6] [13] [15] [21] [25]. These consequences result not only from a loss of trust in the medical field, but also from a sense of personal failure. Women who undergo caesarean sections often recount this sense of failure, which can jeopardize their confidence in their ability to carry another pregnancy [21]. An Italian study showed that $6 \%$ of women listed abuse in childbirth as the main reason for refusing a second pregnancy [6]. The 
fear of being subjected to disrespect and abuse again drives women to plan unattended home births and reduce formal obstetric care [14] [25].

In addition to a loss of self-confidence in their ability to care for their child, some women associate their delivery to sexual assault and rape because of the bodily harm being imposed on them by perpetrators with physical and emotional power over them [6] [11] [14] [21] [32]. Because of the nature of obstetric and gynecologic care, certain acts performed in childbirth can be interpreted as sexual violence by patients, with or without intent from the provider. According to the American College of Obstetricians and Gynecologists (ACOG), acts of sexual misconduct range from "sexual impropriety (behavior, gestures, or expressions that are seductive, sexually suggestive, disrespectful of patient privacy, or sexually demeaning to a patient), including subtle things like neglecting to employ disrobing or draping practices" to rape and sexual assault [33]. ACOG found that less than $10 \%$ of victims report incidents of sexual misconducts, that $100 \%$ of perpetrators are men, and that violent acts occur more often (85\%) in the absence of a chaperone in the room [33].

The notion of guilt that is present in many rape victims' testimonies can be found in women recounting their inability to control their birth. Studies report a high risk of depression and post-traumatic stress disorder (PTSD) in post-partum period. Partners of delivering women can also suffer from psychological consequences and experience similar sentiments of guilt for their inability to protect their partners against harm [6] [14] [21].

Obstetric violence is particularly harmful to women of low socio-economic status (SES), in all regions of the world. For example, one Mexican study found that women delivering in state hospitals had a 3.66 times higher risk of experiencing OV compared to women delivering in private facilities [2]. Another study reported that Mexican midwives commonly witnessed harmful and unnecessary medical procedures, such as manual revisions of the Uterine Cavity, defined as "clearly harmful and ineffective" by WHO, being performed in public hospitals on women without private insurances [12].

Medical students also reported having more freedom to perform multiple pelvic examinations as learning opportunities in public hospitals than in private ones [12]. In the U.S., having private insurance was correlated with lower rates of OV, and women with low SES reported being threatened or shouted at by physicians twice as much as women with moderate and high SES [25]. The absence of private insurance influenced physician's behavior towards women, but also increased women's submissive behavior and their perception of inferiority [7].

Women in minority groups are at higher risk of obstetric violence. A U.S. study reported that $32.8 \%$ of indigenous women had suffered mistreatment by healthcare workers, making them the most likely minority group to experience violence, followed by $25 \%$ of Hispanic women, and $22.5 \%$ of Black women, as compared to $14.1 \%$ of White women [25]. Having a Black partner was also a factor that increased mistreatment. In South America, studies show higher rates 
of mistreatment among women with less education and living in rural settings, as well as unmarried women [2]. In Sri Lanka, Tamil women reported being unable to understand the information provided to them because of the language difference, and being discriminated against because of their minority status [15]. The discrimination that occurs in obstetric care is a reflection of the discrimination women experience in society at large, underlining the structural aspect of obstetric violence (OV) [12] [15].

Power imbalances between physicians and patients reportedly led to increased rates of obstetric violence, especially when disagreement about the delivery occurred, with $78.8 \%$ of women reporting mistreatment when they had different opinions than their health provider on their delivery in a U.S. study [25]. Patients with unexpected procedures such as caesarean sections and women with newborns with health problems were also at higher risk of OV in the U.S. and Mexico [7] [25].

Institutionalized medical practice has legitimized the power dynamics between physicians and patients, as patients often feel entirely dependent on the healthcare provider for current and future care [15]. These power dynamics were reinforced in certain cultures, as exemplified by a Sri Lankan study, where the cultural understanding of who deserved healthcare often justified violence as "Karma", or fate not clear sentence [15].

Obstetric violence is intrinsically related to institutional violence against women. Although the perpetrator is not always male, the victim is always female [23]. When providing a broader context to the occurrence of obstetric violence, many authors report that behaviors towards women in childbirth reflect overall perception of women in broader society, with prevailing patriarchal views that transform the laboring body into a disabled body requiring assistance [3] [7] [12] [21] [24] [29] [32].

The notion of protection of fetal life plays a big role in obstetric violence, as a coercive measure that allows physicians to disregard patient consent, and a legal tool that protects physicians by placing their intent in preserving the baby's safety and any acts done to the mother as justifiable in that intent [23] [30]. Mothers who do not consent to certain acts or offer alternatives to their physician's recommendations are perceived as guilty of not putting the baby's safety first. Mothers' fears and potential lack of health literacy make it easier for physicians to force compliance, using guilt and heightened danger, exemplified by phrases such as "your baby will die if you refuse this", to impose physical acts on patients [25] [32]. The idea that the sole objective of birth is to have a healthy baby ignores women's agency, bringing them back to the status of "(now empty) vessels" [25].

In the United States, some authors tied the concept of protecting fetal life to the debate around abortion. One particular survey found that "physician's personal values ascribed to the fetus (i.e. anti-abortion or conservative attitudes) correlated strongly with their willingness to seek a court-ordered caesarean surgical delivery over the protest of an unwilling patient" [23]. Courts have used 
Roe v. Wade's state interest in protecting fetal life to outweigh a mother's right to choose a birth method [11]. The legal implications of calling to Roe v. Wade to protect physicians' actions fits in the broader context of gender-based violence, by suggesting that women choosing maternal autonomy have an intent to harm or end their baby's life, forcing them "in proxy wars between those who place a premium on maternal autonomy rights and those who believe that fetal interests are more compelling" [23].

Many authors put forward the concept of hyper-medicalizing the birthing process as an enabling factor for obstetric violence, by allowing physicians to justify even unnecessary medical interventions as part of the delivery [12]. This contributed to physicians protesting criminalization of OV, which they perceived as infringing upon medical practice [12] [22]. One author warns about the difference between medicalization and hyper-medicalization. Medicalization refers to "the gradual redefinition of bodily processes as medical domains" [12], which certain authors already see as an abuse of the laboring body that does not require medical attention in the absence of complications [12]. Hyper-medicalization refers to the "overuse or misuse of medicine and technology in health care" [12]. At the center of the discussion around OV is therefore this dichotomy between medicalization and hyper-medicalization, and which medical acts are justified in which context [21] [22].

The environment in which health care professionals operate has also been put forward as enabling incidence of OV in many studies [5] [14] [15] [29] [34]. Most physicians describe their working conditions as precarious, stressful, and inadequate, with low resources, long shifts, and limited staff in obstetrical wards, justifying disrespect and abuse as a result of the pressure endured at work. In Mozambique, a study showed lower rates of obstetric violence in private facilities with better work conditions than in public hospitals [5]. In a study conducted in Sri Lanka, providers described their work conditions as disrespectful and abusive, appropriating the term usually used to describe acts of obstetric violence to describe the health system in which they operate [15]. In Mexico, doctors also reported that the expectation to deliver as many births as possible in a limited time encouraged them to perform cesareans to reach the numbers for financial and administrative gains [14].

The dynamics observed between patients and providers in obstetric violence are often mirrored within the health profession. High levels of sexism and sexual harassment in the medical field are reported in France with $86 \%$ of interns having suffered sexism, and in the United States, with one in three female physicians reporting having experienced sexual harassment in their medical career [6]. Power hierarchies are also observed within the medical field, with a reported imbalance of power between nurses and midwives and physicians. A study performed in Mozambique found that countries with colonial roots tended to have a strong hierarchical relationship between patients and providers, and within the medical setting between physicians and nurses [5]. In turn, the nurses' perceptions of lack of power by nurses within their workspace contribute to increasing 
their need to dominate and control patients [5].

\section{Solutions}

Several authors suggest broadening the role of midwives in birth and delivery as a solution to obstetric violence. In South America, midwives have started a movement to "humanize birth" [12] and return to the roots of midwifery to support laboring women. This is seen as a potential remedy to the hyper-medicalization of birth, and a way to balance out power dynamics by putting women as central decision-makers in their birth, also known as participatory medicine [12] [14]. In the United States, doulas and birth workers act as advocates and witnesses in the delivery room.

Because of the roots of OV in GBV, certain authors address the need for anchoring the debate around obstetric violence in the broader debate around violence against women. As one author puts it, "If recommendations continue to focus on making medical practice more humanized in a non-humanized violent society, the context remains problematic" [21]. Solutions to OV must therefore not be confined to a specific situation in a specific context, but rather to the broader social, economic, and cultural environments in which women operate outside of pregnancy. This is particularly relevant for women in minority groups [7] [12] [21] [22] [30].

To address some of the components that lead to obstetric violence, holistic training should be made available to health professionals, including training on implicit bias, race and culturally competent care [6] [9] [11] [25].

A high volume of Latin American literature and the resulting criminalization of $\mathrm{OV}$ in the region suggest the academic exploration of $\mathrm{OV}$ is conducive to comprehension and concrete measures against it. Criminalization has been presented as a solution to tackle obstetrical violence, but has proven particularly complex, especially where disrespect and abuse are not defined in the law. Apart from South America, where obstetric violence was enacted in the statute on $\mathrm{Vi}$ olence Against Women in 2009, there is a general lack of consensus on the definition of obstetric violence (OV), and because of the knowledge imbalance that often takes place between patients and providers, physicians are rarely prosecuted for incidences of violence [11] [23] [29].

Lawsuits for battery in healthcare are common when patients are "subjected to unconsented touching" [23], but this rarely applies for pregnant women, where the notion of "obstetric logic" [21] is often put forward by physicians to justify potentially violent behavior [11] [21] [23]. To address this gap in criminalization of obstetric violence, one author suggests separating obstetric violence from battery, and instead starting with tort litigations, which would also lengthen the statutes of limitation [23].

Although the knowledge imbalance between patients and physicians makes prosecution hard to achieve, certain authors promote reinforcing accountability mechanisms by empowering both patients and members of the medical team to 
report incidents of OV [6] [9] [11] [12] [25] [29]. Performance indicators of healthcare workers attached to federal funding could also help to "standardize proper care" [4] and encourage respectful behavior [4] [15] [23] [25] [29].

All studies showed that the presence of a witness in the delivery room decreased incidences of violence. In Mozambique, $79.47 \%$ of women interviewed expressed a preference for having their husband in the delivery room [5]. The role of a birth partner during delivery should be further explored and tailored to cultural specificities [5]. In low resource settings, cheap and quick fixes could decrease incidents of $\mathrm{D} \& \mathrm{~A}$, such as curtains between delivery beds in order to encourage privacy [30].

\section{Strength, Limitations, and Future Directions for Research}

The strength of this review is identifying through the current literature that disrespect and abuse in childbirth or obstetric violence occurs worldwide. The main limitation in fully understanding the worldwide prevalence and specific details of OV comes from limitations in the methodology of current publications. Timing of studies can have a significant impact on patient reporting and can lead to bias. A 2016 study in Tanzania showed that when studies were conducted in the medical facility right after birth, $15 \%$ of women reported OV. However, during community interviews performed months later at the patient's house, $70 \%$ of women reported OV. This may be due to a fear of retaliation when answering positively in affirmative for OV in a health facility, and a wish to put the traumatic event that just occurred behind them [3] [4] [13].

Studies are also often focused on a particular region and centered on women's most recent delivery, to avoid recall bias. This may leave out potential incidences of violence from previous births [2]. Moreover, because a clear and standardized definition of OV has not been clarified in every country, certain behaviors have been normalized and may thus not be reported in studies [2] [3] [4] [5] [29]. Women with higher education may be more likely to recognize and name obstetric violence, and thus could mistakenly appear as a high-risk population [5]. Qualitative studies are therefore encouraged to analyze OV given the absence of objectivity. As in other domains of medicine, careful documentation of patient-reported outcomes could lead to better insights from patient about the occurrence of $\mathrm{OV}$ and the effectiveness of interventions [35].

\section{Conclusion}

Most studies focus on the patient perspective, with few providers interviewed. More studies should be conducted to explore the provider perspective on obstetric violence, risk factors for its occurrence, and the context of behavior relative to training and work conditions. The causes of obstetrical violence may be embedded in the structural violence of societies that allow disrespectful and abusive behavior toward women to be normative. A full understanding of this ubiquitous behavior may lead to better support for providers in the form of supplies, 
resources, and back up. Insights into the roots of OV could lead to the development of training programs to reduce its occurrence.

\section{Disclosures}

Support for this project comes from the Gender-Based Violence Fund of Massachusetts General Hospital, Fund number 1200024529.

\section{Conflicts of Interest}

The authors declare no conflicts of interest regarding the publication of this paper.

\section{References}

[1] Bowser, D. and Hill, K. (2010) Exploring Evidence for Disrespect and Abuse in Facility-Based Childbirth: Report of a Landscape Analysis. Harvard School of Public Health and University Research, Washington DC.

[2] Castro, R. and Frías, S.M. (2020) Obstetric Violence in Mexico: Results from a 2016 National Household Survey. Violence Against Women, 26, 555-572. https://doi.org/10.1177/1077801219836732

[3] Abuya, T., Warren, C.E., Miller, N., Njuki, R., Ndwiga, C., Maranga, A., et al. (2015) Exploring the Prevalence of Disrespect and Abuse during Childbirth in Kenya. PLoS $O N E, 10$, e0123606. https://doi.org/10.1371/journal.pone.0123606

[4] Asefa, A. and Bekele, D. (2015) Status of Respectful and Non-Abusive Care during Facility-Based Childbirth in a Hospital and Health Centers in Addis Ababa, Ethiopia. Reproductive Health, 12, Article No. 33.

https://doi.org/10.1186/s12978-015-0024-9

[5] Galle, A., Manaharlal, H., Cumbane, E., et al. (2019) Disrespect and Abuse during Facility-Based Childbirth in Southern Mozambique: A Cross-Sectional Study. BMC Pregnancy and Childbirth, 19, Article No. 369. https://doi.org/10.1186/s12884-019-2532-Z

[6] Haut Conseil à l'Egalité Entre les Femmes et les Hommes (2018) Les actes sexistes durant le suivi gynécologique et obstétrical. Rapport n 2018-06-26-SAN-034.

[7] Santiago, R.V., Monreal, L.A., Rojas Carmona, A. and Domínguez, M.S. (2018) "If We're Here, It's Only Because We Have No Money..." Discrimination and Violence in Mexican Maternity Wards. BMC Pregnancy and Childbirth, 18, Article No. 244. https://doi.org/10.1186/s12884-018-1897-8 https://bmcpregnancychildbirth.biomedcentral.com/track/pdf/10.1186/s12884-018$\underline{1897-8}$

[8] Sethi, R., Gupta, S., Oseni, L., Mtimuni, A., Rashidi, T. and Kachale, F. (2017) The Prevalence of Disrespect and Abuse during Facility-Based Maternity Care in Malawi: Evidence from Direct Observations of Labor and Delivery. Reproductive Health, 14, Article No. 111. https://doi.org/10.1186/s12978-017-0370-x

[9] Shalowitz, D.L. (2020) Sexual Misconduct: ACOG Committee Opinion, Number 796. Obstetrics \& Gynecology, 135, e43-e50. https://doi.org/10.1097/AOG.0000000000003608

[10] Tunçalp, Ö., Were, W.M., MacLennan, C., Oladapo, O.T., Gülmezoglu, A.M., Bahl, R., Daelmans, B., Mathai, M., Say, L., Kristensen, F., Temmerman, M. and Bustreo, F. (2015) Quality of Care for Pregnant Women and Newborns-The WHO Vision. 
BJOG: An International Journal of Obstetrics \& Gynaecology, 122, 1045-1049. https://doi.org/10.1111/1471-0528.13451

[11] Borges, M.T. (2018) A Violent Birth: Reframing Coerced Procedures during Childbirth as Obstetric Violence. Duke Law Journal, 67, 827-862.

[12] Dixon, L.Z. (2015) Obstetrics in a Time of Violence: Mexican Midwives Critique Routine Hospital Practices. Medical Anthropology Quarterly, 29, 437-454.

[13] Sando, D., Ratcliffe, H., McDonald, K., Spiegelman, D., Lyatuu, G., Mwanyika-Sando, M., Emil, F., Wegner, M.N., Chalamilla, G. and Langer, A. (2016) The Prevalence of Disrespect and Abuse during Facility-Based Childbirth in Urban Tanzania. BMC Pregnancy and Childbirth, 16, 236.

[14] Gonzales-Flores, M. (2015) Resistance of Mayan Women against Obstetric Violence. Global Societies Journal, 3, 1-11. https://escholarship.org/uc/item/24g728jb

[15] Perera, D., Lund, R., Swahnberg, K. et al. (2018) 'When Helpers Hurt': Women's and Midwives' Stories of Obstetric Violence in State Health Institutions, Colombo District, Sri Lanka. BMC Pregnancy Childbirth, 18, 211.

[16] Centers for Disease Control and Prevention (2018) Health-Related Quality of Life. https://www.cdc.gov/hrqol/concept.htm

[17] Nijagal, M.A., Wissig, S., Stowell, C., Olson, E., Amer-Wahlin, I., Bonsel, G., Brooks, A., Coleman, M., et al. (2018) Standardized Outcome Measures for Pregnancy and Childbirth, an ICHOM Proposal. BMC Health Services Research, 18, Article No. 953. https://doi.org/10.1186/s12913-018-3732-3

[18] Srivastava, A., Avan, B.I., Rajbangshi, P. and Bhattacharyya, S. (2015) Determinants of Women's Satisfaction with Maternal Health Care: A Review of Literature from Developing Countries. BMC Pregnancy and Childbirth, 15, Article No. 97. https://doi.org/10.1186/s12884-015-0525-0

[19] Wall, L.L. (2006) The Medical Ethics of Dr. J Marion Sims: A Fresh Look at the Historical Record. Journal of Medical Ethics, 32, 346-350.

https://doi.org/10.1136/jme.2005.012559

[20] Cheyney, M. and Davis-Floyd, R. (2019) Birth in Eight Cultures: Brazil, Greece, Japan, Mexico, the Netherlands, New Zealand, Tanzania, United States. Waveland Press, Long Grove.

[21] Murray de Lopez, J. (2018) When the Scars Begin to Heal: Narratives of Obstetric Violence in Chiapas, Mexico. International Journal of Health Governance, 23, 60-69. https://doi.org/10.1108/IJHG-05-2017-0022

[22] Macias, A. (2019) Perception du débat sur les violences obstétricales par les professionnels, sages-femmes et gynécologues-obstétriciens. Gynécologie et obstetrique, ffdumas-02301776.

[23] Diaz-Tello, F. (2016) Invisible Wounds: Obstetric Violence in the United States. Reproductive Health Matters, 24, 56-64. https://doi.org/10.1016/j.rhm.2016.04.004

[24] Vacaflor, C.H. (2016) Obstetric Violence: A New Framework for Identifying Challenges to Maternal Healthcare in Argentina. Reproductive Health Matters, 24, 65-73. https://doi.org/10.1016/j.rhm.2016.05.001

[25] Vedam, S., Stoll, K., Taiwo, T.K., et al. (2019) The Giving Voice to Mothers Study: Inequity and Mistreatment during Pregnancy and Childbirth in the United States. Reproductive Health, 16, Article No. 77. https://doi.org/10.1186/s12978-019-0729-2

[26] Whittemore, R. and Knafl, K. (2005) The Integrative Review: Updated Methodology. Journal of Advanced Nursing, 52, 546-553. https://doi.org/10.1111/j.1365-2648.2005.03621.x 
[27] Torraco, R.J. (2005) Writing Integrative Literature Reviews: Guidelines and Examples. Human Resource Development Review, 4, 356-367. https://doi.org/10.1177/1534484305278283

[28] World Health Organization (2020) Sexual and Reproductive Health: Prevention and Elimination of Disrespect and Abuse during Childbirth.

https://www.who.int/reproductivehealth/topics/maternal_perinatal/statement-child birth-data/en/

[29] Jardim, D. and Modena, C.M. (2018) Obstetric Violence in the Daily Routine of Care and Its Characteristics. Revista Latino-Americana de Enfermagem, 26, e3069. https://doi.org/10.1590/1518-8345.2450.3069

[30] Bohren, M.A., Mehrtash, H., Fawole, B., Maung, T.M., Balde, M. D., Maya, E., Tunçalp, Ö., et al. (2019) How Women Are Treated during Facility-Based Childbirth in Four Countries: A Cross-Sectional Study with Labour Observations and Community-Based Surveys. The Lancet, 394, 1750-1763.

https://doi.org/10.1016/S0140-6736(19)31992-0

[31] Ghanbari-Homayi, S., Fardiazar, Z., Meedya, S., et al. (2019) Predictors of Traumatic Birth Experience among a Group of Iranian Primipara Women: A Cross Sectional Study. BMC Pregnancy Childbirth, 19, Article No. 182.

https://doi.org/10.1186/s12884-019-2333-4

[32] Cohen Shabot, S. (2016) Making Loud Bodies "Feminine": A Feminist-Phenomenological Analysis of Obstetric Violence. Human Studies, 39, 231-247. https://doi.org/10.1007/s10746-015-9369-x

[33] ACOG (2020) Sexual Misconduct. Obstetrics \& Gynecology, 135, e43-e50. https://doi.org/10.1097/AOG.0000000000003608

[34] Sando, D., Abuya, T., Asefa, A., et al. (2017) Methods Used in Prevalence Studies of Disrespect and Abuse during Facility-Based Childbirth: Lessons Learned. Reproductive Health, 14, Article No. 127. https://doi.org/10.1186/s12978-017-0389-Z

[35] Mahmud, A., Morris, E., Johnson, S. and Ismail, K.M. (2014) Developing Core Patient-Reported Outcomes in Maternity: PRO-Maternity. BJOG: An International Journal of Obstetrics \& Gynaecology, 121, 15-19.

https://doi.org/10.1111/1471-0528.12901 


\section{Abbreviations}

D \& A: Disrespect and Abuse

OEC: Observatory of Economic Complexity

OV: Obstetric Violence

PTSD: Posttraumatic Stress Disorder

SES: Socioeconomic Status

VAW: Violence Against Women

WHO: World Health Organization 